\title{
Dynamic Stability of a Thin Plate Subjected to Bi-Axial Edged Loads
}

\author{
Abbas Talimian, Péter Béda \\ Department of Vehicle Elements and Vehicle-Structure Analysis, Budapest \\ University of Technology and Economics, Múegyetem rakpart 3, 1111 Budapest, \\ Hungary; talimian@kme.bme.hu, bedap@kme.bme.hu
}

\begin{abstract}
The first dynamic stability of a thin rectangular plate made from linear elastic material and subjected to a bi-axial time dependent load is discussed in this study. The plate carries a combination of loads that are changing linearly with respect to the length of its edges. It is also supposed that its boundary conditions on the edges are simply supported. Relevant inertia, elastic stiffness, and stability matrices are derived by applying Finite Difference Method from a differential motion equation of the plate. Subsequently, Mathieu-Hill equation form is obtained for the plate and the first dynamic stability boundaries of the plate are elaborated for using specified load parameters.
\end{abstract}

Keywords: rectangular plate; Finite Difference Method (FDM); bi-axial load; Dynamic stability; Mathieu-Hill equations

\section{Introduction}

Thin plates are used in several structures as elements for different applications such as aerospace, civil structures, containers, ships, and machinery parts. These elements are subjected to various loading types, e.g. statics, dynamic and thermal as well. Sometimes these loads are reactions from other elements. The present study models a situation when a rectangular plate as a structure's element is subjected to loads acting on its edges. Loads are in-plane and vary along the length of the edge as a general condition. Loads' effect on an element behaviour is crucial, especially when these parts are subjected to time-dependent loading. In the designing procedure of a machine's elements, determining buckling load (static stability) and doing dynamic stability analysis can give a better overview of either a structure or its elements while it is loaded with different force types. Up to now, numerous literature has been discussed concerning these fields of study.

A rectangular plate with localised zones of damage and loaded by either uni- or biaxial compressive uniform edge load(s) was considered by Prabhakara and Datta for studying the free vibration and static stability analysis by application of finite 
element analysis. The plate was discussed while parameters such as damage size, its position, boundary condition and aspect ratio were changing [1]. They worked on the tension and compression buckling of a square plate with localised zones of damage and loaded partially on its edge by using finite element method. A plate stability was discussed with respect to damage location and its size as well as the position of load [2]. Their study was extended for a plate with internal opening while in-plane compressive or periodic tensile loading acted on the plate's edge. The study was done for different shapes and cut-out sizes [3].

Deolasi and Datta studied a plate subjected to localised in-plane either compressive or tensile periodic edge loads for finding buckling loads and parametric instability analysis. The transverse shear deformation and rotary inertia were considered as well. The load type, its band and location effect on vibration and parametric instability were elaborated [4]. They also applied finite element method to obtain the equilibrium equation of a simply supported rectangular plate subjected to partially in-plane periodic loading for investigating the damping effect on the behaviour of simple and combination resonances. The method of multiple scales was applied for obtaining boundaries of parametric instability region's relations [5].

A research was done on a rectangular plate with completely elastically restrained boundaries by application of the variational method for governing equations. The plate rested on the non-homogeneous foundation as well and was subjected to a uniform compressive in-plane bi-axial time-dependent load. The foundation has two regions with different stiffness, but symmetric about the centre lines of the plate. The method of multiple scales was applied for analysing Mathieu-Hill equations and elaborating stability boundaries [6]. Sahu and Datta used the first order shear deformation theory for modelling the doubly curved panels to consider the effects of transverse shear deformation and rotary inertia. They used finite element analysis application for obtaining parametric instability characteristics of the panels. It was supposed loads on edges are various in-plane static and periodic compressive, including partial and concentrated type [7]. The study was continued for studying parametric instability behaviour of curved panels with cut-outs with in-plane static and periodic compressive edge loadings [8]. Kumar et al. investigated vibration and dynamic instability behaviour of laminated composite plates subjected to partially distributed non-conservative follower forces and uniaxial in-plane point and patch tensile edge loadings by consideration of finite element method and first order shear deformation theory for modelling laminated composite plates and doubly curved panels [9, 10]. Dynamic instability analysis was done for stiffened plates carried in-plane partial and concentrated edge loadings by Srivastava et al. The plate and the stiffeners were modelled as separate elements and the compatibility between these two types of elements is maintained [11]. Tensile buckling, vibration, and parametric instability behaviour of doubly curved panels with central circular cut-out regarding uniaxial in-plane partially distributed tensile edge loadings were studied by Ravi Kumar et al. [12]. They 
also expanded their research to laminated composite doubly curved panels, subjected to non-uniform follower load. Their formulation was based on the extension of dynamic, shear deformable theory according to Sanders' first approximation for doubly curved laminated shells [13]. Static and dynamic instability analysis were done for stiffened shell panels with a uniform in-plane harmonic edge by Patel et al. [14]. They used eight-node isoparametric degenerated shell element for shell panels and three-node curved beam element for stiffeners.

Whenever the loading types are not constant or a structure is complex, solving the partial differential equation of motion will be impossible from analytical methods point of view and a numerical solution technique such as finite element method has to be applied to estimate a solution and studying free vibration and static stability analysis of thin isotropic plates $[15,16]$.

There are also some suggestions on the basis of either exact or numerical method for seeking a solution for deriving the partial differential equation of motion. The majority of these studies focused on either uniaxial constant in-plane loads or pressure acts perpendicular to the plate face leading to a constant coefficient differential motion equation (i.e. some of the most recent studies are [17-21]). The buckling load was derived by the help of Differential Quadrature Method (DQM) for a thin rectangular plate while it was loaded by either uni- or bi-axial concentrated loads meanwhile different combination of simply supports and clamped boundary conditions were involved [22].

Another possible numerical method for estimating solutions is the Finite Difference Method (FDM), which is based on replacing differential equations with corresponding difference equations (see [23, 24]). Using FDM makes solving the differential form of motion equation straightforward. Werfalli and Karoud studied free vibration of thin isotropic rectangular plates having various boundary conditions [25]. They used a Galerkin-based finite element method for deriving a mathematical model represents the vibration behaviour of the plate. Cubic quadrilateral serendipity elements with twelve degrees of freedom were considered in their analysis. An available Modified Discrete Kirchhoff Quadrilateral (MDKQ) element that is developed based on Classical Plate Theory (CPT) by using discrete Kirchhoff technique is applied by Patil in free vibration response analysis of a thin isotropic rectangular plate [26]. This method had just considered only for static analysis of plate formerly. It was also tried to assess the accuracy of the developed finite element formulation in analysing the vibration response of a thin isotropic rectangular plate that has different boundary conditions and geometrical dimensions ratios. An Ordinary Finite Difference Method (OFDM), was applied in pure bending and free vibration analysis of a thin flat rectangular plate by Ezeh, et al. [27, 28].

To our best knowledge, there has been no study for considering the influence of linear bi-axial loading effect on the dynamic stability of a thin plate. 
A Ph.D. dissertation was written on the investigation of parametric instability of thin plates [29]. The motion equation of the plate was derived on basis of the first order shear theory by using the FEM. However, the author just discussed the axial loading effect on the dynamic stability regions. Here, we have a thin plate as a simplification model. It is supposed the displacement field is continuous and its derivatives exist for sufficient times, hence applying FDM helps to derive inertia, elastic stiffness and stability matrices for the plate from a differential motion equation easily. As a consequence, the dynamic stability analysis is done for linear bi-axial loading.

\section{Problem Formulation}

Here a thin rectangular plate that is made from a linear elastic material is considered. $a$ and $b$ are plate's width and height along the $x_{1^{-}}$and $x_{2^{-}}$axes respectively, Figure 1. $h$ is the thickness of the plate along the $x_{3^{-}}$axis. While a thin plate is selected for the study, it is supposed the ratio of thickness to the smallest dimension is lower than five percent $(h / b \leq 0.05)$. The plate is subjected to different linearly in-plane bi-axial loads on its edges as shown in Figure 2. The following assumptions, which are fundamental points in the small-deflection plate theory, are valid here as well [24].
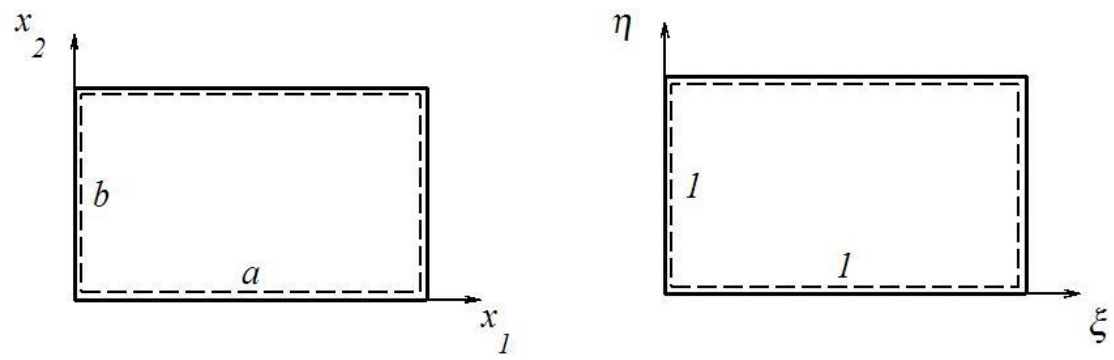

Figure 1

Plate Dimensions

It is supposed that: (1) There is no deflection in the plate's mid-surface in comparison with its thickness and, consequently, the square of the slope can be omitted compared to unity. (2) Plane sections normal to mid-surface initially remain plane and normal to the surface after bending. (3) The translation's component along to the normal axis to the mid-surface is independent of $x_{3^{-}}$.

The differential motion equation of a thin plate subjected to bi-axial in-plane load is given by $[24,30]$, 


$$
D \nabla^{4} \tilde{w}+\rho h \frac{\partial^{2} \tilde{w}}{\partial t^{2}}=q+N_{x_{1}} \frac{\partial^{2} \tilde{w}}{\partial x_{1}^{2}}+2 N_{x_{1} x_{2}} \frac{\partial^{4} \tilde{w}}{\partial x_{2}^{2} \partial x_{1}^{2}}+N_{x_{2}} \frac{\partial^{2} \tilde{w}}{\partial x_{2}{ }^{2}} .
$$

where $\rho$ represents density per unit volume and time is given by $t$. Normally distributed load per unit area on the plate surface, in-plane forces per unit length acting on plate's edges in the direction of $x_{1^{-}}$and $x_{2^{-}}$axes are $q, N_{x_{1}}, N_{x_{2}}$ respectively. The shearing force per unit length in the $x_{1} x_{2}$ - the plane is $N_{x_{1} x_{2}}$. The Flexural rigidity of a plate is $E$ and $v$ are Young's modulus and Poisson's ratio respectively. $\tilde{w}$, is the time-dependent displacement, with angular speed of $\omega$, along the axis perpendicular to the plate's plane,

$\tilde{w}=w\left(x_{1}, x_{2}, t\right)$.

Assume that there is no shear load $\left(N_{x_{1} x_{2}}=0\right)$ and normally distributed load $(q=0)$ acting on the plate. In-plane forces $\left(N_{x_{1}}\right.$ and $\left.N_{x_{2}}\right)$ acting on the plate's edges that varying along a length, are:

$$
N_{x_{1}}=-N_{0}\left(1-\frac{\alpha x_{2}}{b}\right), \quad N_{x_{2}}=-N_{0}\left(1-\frac{\beta x_{1}}{a}\right) .
$$

In-plane load intensity is $N_{0} . \alpha$ and $\beta$ are selected numbers $(\alpha, \beta=\{1,2\})$ and are called load parameters. The possible bi-axial loading is given in Figure 2. Substituting (2) and (3) to the differential motion equation of plate (1) gives the following equation

$$
D \nabla^{4} w+\rho h \frac{\partial^{2} w}{\partial t^{2}}+N_{0}\left(1-\frac{\alpha x_{2}}{b}\right) \frac{\partial^{2} w}{\partial x_{1}^{2}}+N_{0}\left(1-\frac{\beta x_{1}}{a}\right) \frac{\partial^{2} w}{\partial x_{2}^{2}}=0,
$$

It is supposed here the plate has simply supported boundary conditions on its edges which prevent transverse deflection and allows rotations. These boundary conditions equations are given as,

$$
\begin{aligned}
& \left\{\begin{array}{l}
w\left(0, x_{2}\right)=0, w\left(a, x_{2}\right)=0, \\
w\left(x_{1}, 0\right)=0, w\left(x_{1}, b\right)=0 .
\end{array}\right. \\
& \begin{cases}\left.\frac{\partial^{2} w\left(x_{1}, x_{2}\right)}{\partial x_{1}^{2}}\right|_{x_{1}=0}=0, & \left.\frac{\partial^{2} w\left(x_{1}, x_{2}\right)}{\partial x_{1}^{2}}\right|_{x_{1}=a}=0, \\
\left.\frac{\partial^{2} w\left(x_{1}, x_{2}\right)}{\partial x_{2}^{2}}\right|_{x_{2}=0}=0, & \left.\frac{\partial^{2} w\left(x_{1}, x_{2}\right)}{\partial x_{2}^{2}}\right|_{x_{2}=b}=0 .\end{cases}
\end{aligned}
$$

The non-dimensional parameters for the horizontal, $\xi$ and vertical axes, $\eta$ (see Figure 1) are introduced as,

$$
x_{1}=\xi a, \quad\left\{\begin{array}{ll}
\text { for } x_{1}=0, & \xi=0 . \\
\text { for } x_{1}=a, & \xi=1 .
\end{array} \text { and } x_{2}=\eta b, \quad \begin{cases}\text { for } x_{2}=0, & \eta=0 . \\
\text { for } x_{2}=b, & \eta=1 .\end{cases}\right.
$$


Hence the equation of motion of the plate (4) is written in non-dimensional form,

$$
\zeta^{2} \frac{\partial^{2} w}{\partial t^{2}}+\left(\frac{\partial^{4} w}{\partial \xi^{4}}+2 r^{2} \frac{\partial^{4} w}{\partial \xi^{2} \partial \eta^{2}}+r^{4} \frac{\partial^{4} w}{\partial \eta^{4}}\right)+\bar{N}\left((1-\alpha \eta) \frac{\partial^{2} w}{\partial \xi^{2}}+r^{2}(1-\beta \xi) \frac{\partial^{2} w}{\partial \eta^{2}}\right)=0 .
$$
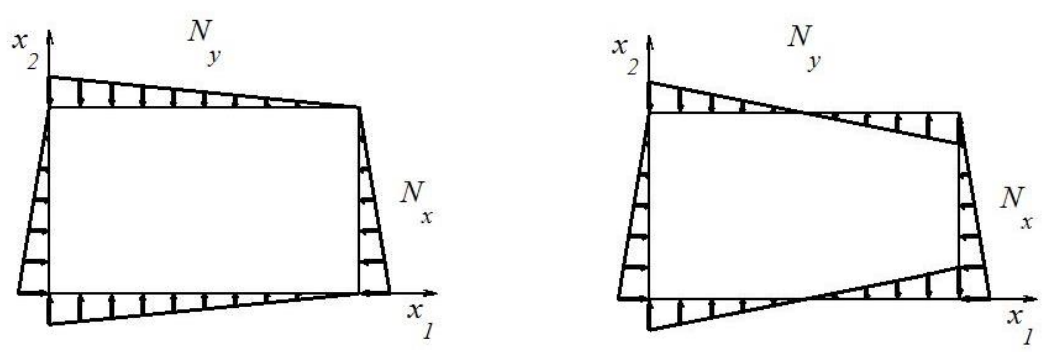

$N_{x_{1}}=-N_{0}\left(1-\frac{x_{2}}{b}\right), N_{x_{2}}=-N_{0}\left(1-\frac{x_{1}}{a}\right)$

$$
N_{x_{1}}=-N_{0}\left(1-\frac{x_{2}}{b}\right), N_{x_{2}}=-N_{0}\left(1-\frac{2 x_{1}}{a}\right) \text {. }
$$
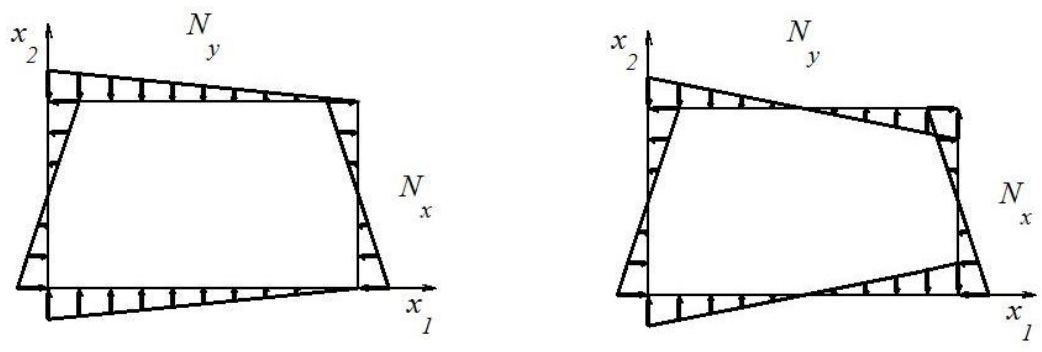

$$
N_{x_{1}}=-N_{0}\left(1-\frac{2 x_{2}}{b}\right), N_{x_{2}}=-N_{0}\left(1-\frac{x_{1}}{a}\right) . \quad N_{x_{1}}=-N_{0}\left(1-\frac{2 x_{2}}{b}\right), N_{x_{2}}=-N_{0}\left(1-\frac{2 x_{1}}{a}\right) .
$$

Figure 2

Loading types

$\mathrm{r}$, is a geometrical aspect that represents the ratio of plate's length over its height. Buckling load and frequency factors are $\bar{N}=N_{0} a^{2} / D$ and $\zeta^{2}=\rho h a^{4} / D$ respectively. Boundary conditions (5) are also given as non-dimension equations,

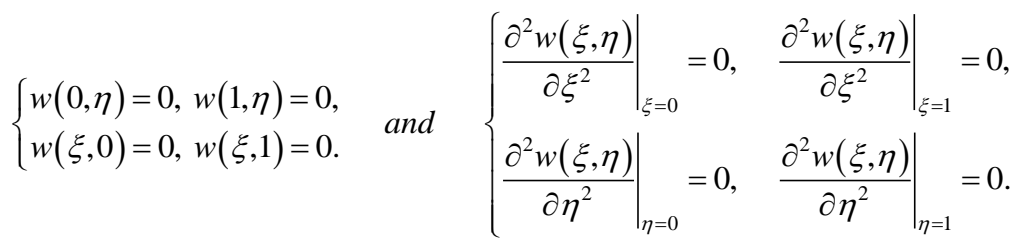




\section{Discretization of the Problem: Finite Difference Method}

Finite difference method as a numerical method is used in solving a differential equation. Here the motion equation of the plate (7) is given by a fourth order differential equation. For approximating each term of it with difference equation on solution's domain the plate's surface is uniformly discretized to $N \times N$ equal sub-domain rectangular meshes. The length and width of each mesh's grid equal to $1 / N$, Consequently, each node's abscissa and ordinate are,

$\begin{cases}\xi_{i}=i / N, & i=1 \ldots N . \\ \eta_{j}=j / N, & j=1 \ldots N .\end{cases}$

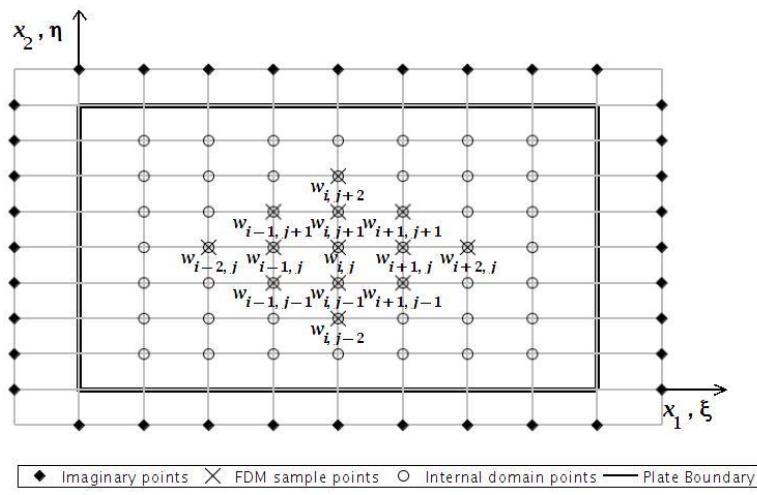

Figure 3

Internal, boundary and imaginary points of a plate

The second and fourth derivatives of displacement field respect to the selected non-dimensional parameters for the horizontal and vertical axes are defined subsequently on the basis of central difference formulas for node $w_{i, j}(\xi, \eta)$ (i.e. $[24,27,28])$,

$$
\begin{aligned}
& \frac{1}{N^{2}}\left(\frac{\partial^{2} w(\xi, \eta)}{\partial \xi^{2}}\right)=\left(w_{i-1, j}+w_{i+1, j}\right)-2 w_{i, j}, \\
& \frac{1}{N^{2}}\left(\frac{\partial^{2} w(\xi, \eta)}{\partial \eta^{2}}\right)=\left(w_{i, j-1}+w_{i, j+1}\right)-2 w_{i, j} .
\end{aligned}
$$




$$
\begin{aligned}
& \frac{1}{N^{4}}\left(\frac{\partial^{4} w(\xi, \eta)}{\partial \xi^{4}}\right)=\left(w_{i-2, j}+w_{i+2, j}\right)-4\left(w_{i-1, j}+w_{i+1, j}\right)+6 w_{i, j}, \\
& \frac{1}{N^{4}}\left(\frac{\partial^{4} w(\xi, \eta)}{\partial \xi^{2} \partial \eta^{2}}\right)=\left(w_{i-1, j+1}+w_{i+1, j+1}+w_{i-1, j-1}+w_{i+1, j-1}\right) \\
& -2\left(w_{i-1, j}+w_{i+1, j}+w_{i, j-1}+w_{i, j+1}\right)+4 w_{i, j}, \\
& \frac{1}{N^{4}}\left(\frac{\partial^{4} w(\xi, \eta)}{\partial \eta^{4}}\right)=\left(w_{i, j-2}+w_{i, j+2}\right)-4\left(w_{i, j-1}+w_{i, j+1}\right)+6 w_{i, j} \text {. }
\end{aligned}
$$

The plate's differential equation of motion (7) terms is replaced with relevant difference equations (10) and (11). Boundary conditions have to be also modified with consideration of (10) and are written as,

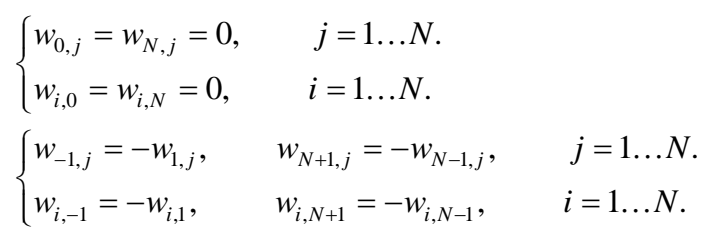

A set of algebraic equations is derived for every internal point of a plate, Figure 3. These equations are collected in a matrix equation,

$\underline{\underline{M}} \underline{\ddot{w}}+(\underline{\underline{K}}+\bar{N} \underline{\underline{S}}) \underline{w}=\underline{0}$.

$\underline{\underline{M}}$, is a diagonal matrix and called either mass or inertia matrix as its elements are mass properties. Elastic stiffness and stability matrices are given by $\underline{\underline{K}}$ and $\underline{\underline{S}}$ respectively. They are band matrices and their elements are computed from second and third group equations in (7). Elastic behaviour of the plate is affected by $\underline{\underline{K}}$ 's elements while loading terms are collected in $\underline{\underline{S}} \cdot \underline{w}$ is a nodal displacement vector contains internal points' displacements of the plate, Figure 3, along with normal axis $x_{3}$ - perpendicular to the mid-surface plane of the plate.

\section{Dynamic Stabiliy Analysis, the Basic Solution}

The plate is subjected to in-plane time-dependent loads on its edges. External loads (3) on the plate's edges are,

$$
N_{0}(t)=N_{s}+N_{d} \cos (\Omega t)
$$

as a function of time. $N_{s}$ and $N_{d}$ are the static and dynamic amplitude of timedependent load respectively. 
$\Omega$ represents an excitation frequency, (14) is simplified in the form of,

$N_{0}(t)=\frac{1}{2} N_{c r 0}(\lambda+\mu \cos (\Omega t))$.

$N_{c r 0}$, represents the critical buckling load and can be calculated by solving the eigenvalue problem (13) for $\bar{N}$ if stability analysis at static loads is considered. Static load factor, $\lambda$, and dynamic load factor, $\mu$, are defined by comparing (14) and (15).

$\lambda=\frac{2 N_{s}}{N_{c r 0}}, \quad \mu=\frac{2 N_{d}}{N_{c r 0}}$.

Substituting in-plane time-dependent load (15) in plate's motion equation(13), one has,

$\underline{\underline{M}} \underline{\ddot{w}}+\left(\underline{\underline{K}}+\frac{1}{2} N_{c r 0}(\lambda+\mu \cos (\Omega t)) \underline{\underline{S}}\right) \underline{w}=\underline{0}$.

The latter equation is Mathieu-Hill [31]. Some methods have been advised for solving these type of equations, as the experimental solutions, i.e. Bolotin monograph, Galerkin, Lyapunov's second method, asymptotic techniques, perturbation and iteration [32-36]. There exists no analytic solution for (17), second order differential equations with a periodic coefficient in general. Hence, an approximated time-dependent periodic function is used as a trial solution based on a method advised and applied in [31] with a $2 T$ period ( a first approximation of the first region of stability) for (17).

$$
\begin{aligned}
& \underline{w}=\sum_{k=1,3,5, \cdots}^{\infty} a_{k} \sin \left(\frac{k \Omega t}{2}\right)+\underline{b_{k}} \cos \left(\frac{k \Omega t}{2}\right), \\
& \ddot{w}=-\left(\frac{k \Omega}{2}\right)^{2} \underline{w} .
\end{aligned}
$$

Introducing (18) into (17) returns the motion equation of the plate as

$$
\begin{aligned}
& \sum_{k=1,3,5, \cdots}^{\infty}\left[-\underline{\underline{M}}\left(\frac{k \Omega}{2}\right)^{2}+\underline{\underline{K}}+\frac{1}{2} \lambda N_{c r 0} \underline{\underline{S}}\right]\left(\underline{a_{k}} \sin \left(\frac{k \Omega t}{2}\right)+\underline{b_{k}} \cos \left(\frac{k \Omega t}{2}\right)\right) \\
& +\frac{1}{2} \mu \cos (\Omega t) N_{c r} \underline{\underline{S}}\left(\underline{a_{k}} \sin \left(\frac{k \Omega t}{2}\right)+\underline{b_{k}} \cos \left(\frac{k \Omega t}{2}\right)\right)=\underline{0} .
\end{aligned}
$$

From the mathematics point of view of trigonometric relations in sine and cosine multiplication (19) can be simplified. (19) is easily separated by sine and cosine terms as far as they are linearly independent mathematical functions. The final form of motion equation of the plate is, 


$$
\begin{aligned}
\sum_{k=1,3,5, \cdots}^{\infty} & \sin \left(\frac{k \Omega t}{2}\right)\left[-\underline{\underline{M}}\left(\frac{k \Omega}{2}\right)^{2}+\underline{\underline{K}}+\frac{1}{2} \lambda N_{c r 0} \underline{\underline{S}}\right] \underline{a_{k}} \\
+ & \frac{1}{4} \mu N_{c r 0} \underline{S}\left[\sin \left((k+2) \frac{\Omega t}{2}\right)+\sin \left((k-2) \frac{\Omega t}{2}\right)\right] \underline{a_{k}} \\
+ & \cos \left(\frac{k \Omega t}{2}\right)\left[-\underline{\underline{M}}\left(\frac{k \Omega}{2}\right)^{2}+\underline{\underline{K}}+\frac{1}{2} \lambda N_{c r 0} \underline{\underline{S}}\right] \underline{b_{k}} \\
+ & \frac{1}{4} \mu N_{c r 0} \underline{S}\left[\cos \left((k+2) \frac{\Omega t}{2}\right)+\cos \left((k-2) \frac{\Omega t}{2}\right)\right] \underline{b_{k}}=\underline{0} .
\end{aligned}
$$

Selecting just first two counters in $(20)(k=1,3)$ to obtain the first dynamic stability regions leads (21). It has to be solved for having a non-trivial solution, hence each matrix's determinant has to be zero. These equations are separated into two independent eigenvalue problems regarding the linear independence of the unknown constant in (18), $\underline{a_{k}}$ and $\underline{b_{k}}$.

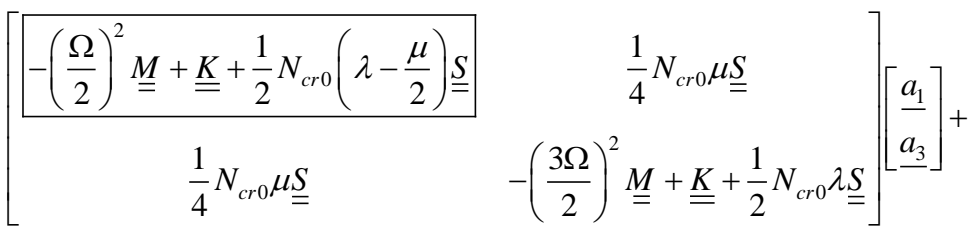

$$
\begin{aligned}
& {\left[\frac{\left[-\left(\frac{\Omega}{2}\right)^{2} \underline{\underline{M}}+\underline{\underline{K}}+\frac{1}{2} N_{c r 0}\left(\lambda+\frac{\mu}{2}\right) \underline{\underline{S}}\right.}{\frac{1}{4} N_{c r 0} \mu \underline{\underline{S}}}-\left(\frac{3 \Omega}{2}\right)^{2} \underline{\underline{M}}+\underline{\underline{K}}+\frac{1}{2} N_{c r 0} \lambda \underline{\underline{S}}\right]\left[\left[\begin{array}{l}
\underline{b_{1}} \\
\underline{b_{3}}
\end{array}\right]=\underline{0} .\right.}
\end{aligned}
$$

The first elements of the eigenvalue problem (22) are just selected for analysing the dynamic stability of the plate and returns the first dynamic stability boundaries. Dynamic stability analysis of a structure, here a thin plate, is closely related to changing in parameters such as $\Omega, N_{c r 0}, \lambda$ and $\mu$. The excitation frequency is supposed to be a ratio of the natural frequency of the plate $(\Omega=\phi \omega)$. Here, $\phi$ is Excited Frequency Ratio. While no load acts on the plate $(\bar{N}=0)$ the natural frequency of the plate, $\omega$, can be calculated from (13).

$$
\begin{aligned}
& \operatorname{det}\left(-\frac{\varphi^{2} \omega^{2}}{4} \underline{\underline{M}}+\underline{\underline{K}}+\frac{1}{2} \bar{N}_{c r 0}\left(\lambda-\frac{\mu}{2}\right) \underline{\underline{S}}\right)=0, \\
& \operatorname{det}\left(-\frac{\varphi^{2} \omega^{2}}{4} \underline{\underline{M}}+\underline{\underline{K}}+\frac{1}{2} \bar{N}_{c r 0}\left(\lambda+\frac{\mu}{2}\right) \underline{\underline{S}}\right)=0 .
\end{aligned}
$$




\section{Results and Calculations}

The first dynamic stability boundaries, show domains where the plate goes to be either stable or lose its stability respect to time-dependent excitation load, are plotted from (22) and illustrated in Figure 4. Plots are drawn here for three different static load factors $(\lambda=\{0.3,0.6,0.9\})$. If effective parameters, $\phi$, and $\mu$, are selected from inside boundaries' region, the plate loses its stability. On the other hand, whenever latter parameters are chosen from these boundaries' outside, the plate's motion regarding assumed load (15) remains stable.
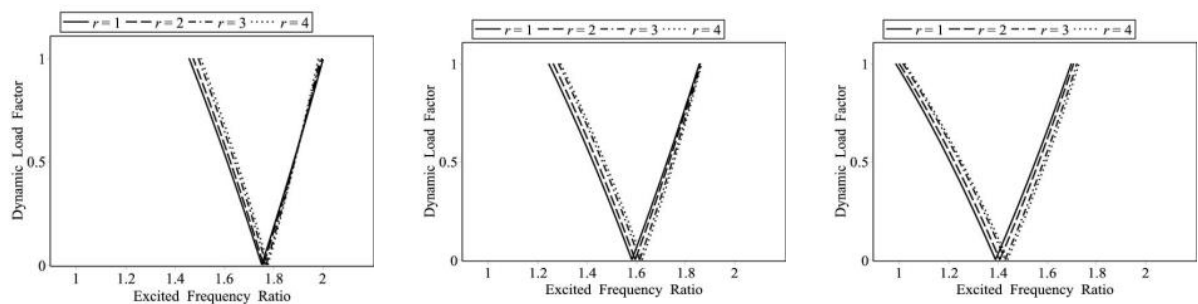

$(\alpha=1, \beta=1, \lambda=0.3)$

$(\alpha=1, \beta=1, \lambda=0.6)$

$(\alpha=1, \beta=1, \lambda=0.9)$
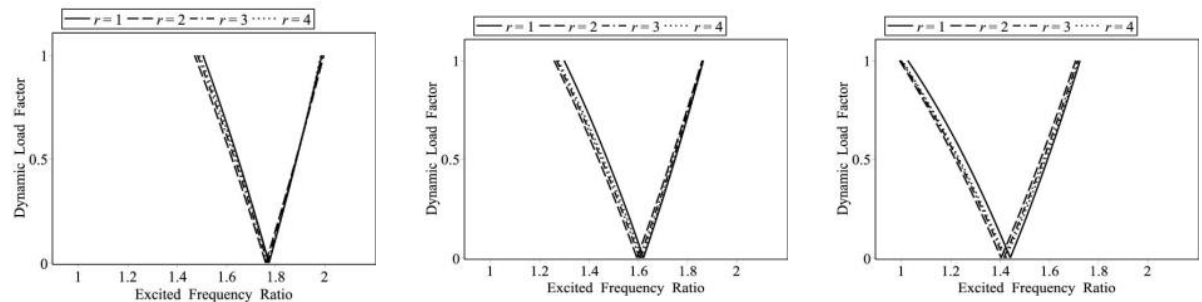

$(\alpha=2, \beta=1, \lambda=0.3)$

$(\alpha=2, \beta=1, \lambda=0.6)$

$(\alpha=2, \beta=1, \lambda=0.9)$
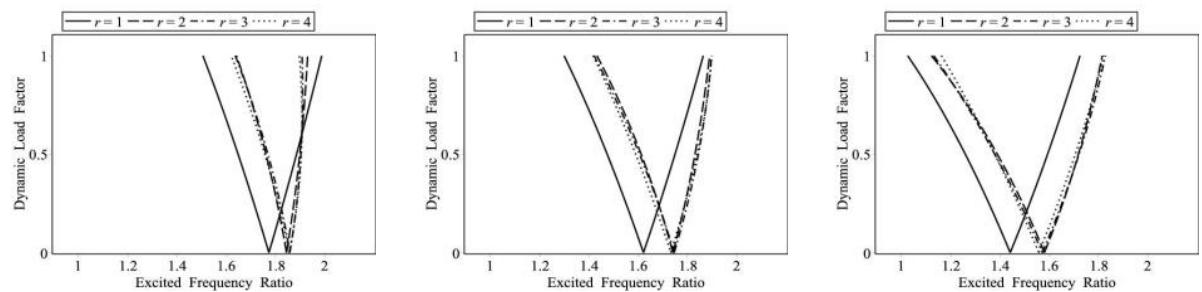

$(\alpha=1, \beta=2, \lambda=0.3)$

$(\alpha=1, \beta=2, \lambda=0.6)$

$(\alpha=1, \beta=2, \lambda=0.9)$ 


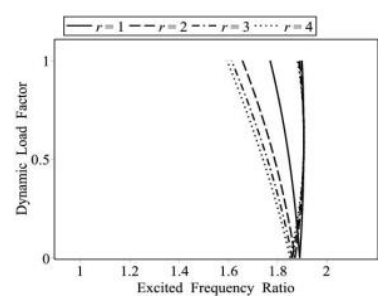

$(\alpha=2, \beta=2, \lambda=0.3)$

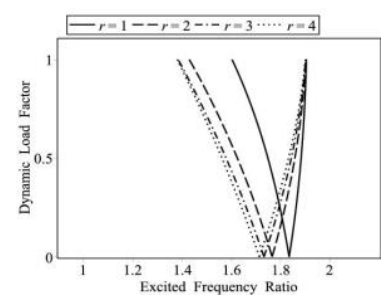

$(\alpha=2, \beta=2, \lambda=0.6)$

Figure 4

Dynamic Stability Diagrams

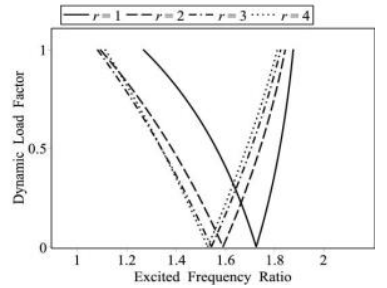

$(\alpha=2, \beta=2, \lambda=0.9)$

\section{Conclusions}

In this study, a thin rectangular plate is made from linear elastic material subjected to bi-axial time dependent loads was supposed. A combination of two in-plane load act on edges, changing linearly respect to the length, was considered. The plate has simply supported boundary conditions on its edges. The plate's displacement field is continuous and can be differentiated sufficient time hence the Finite Difference Method is applied for solving the fourth order differential equation of the motion. The first dynamic stability boundaries of the plate respect to load parameters and static load factor are illustrated in Figure 4. According to these results, following points can be obtained.

Increasing the share of the static load factor $\lambda$ causes the first stability range of the plate regardless any combination of load types being decreased (borders' gap are going to be wider).

Generally, the plate loses its stability around lower excited frequency ratios whenever greater numbers are selected for the static load factor.

Whenever in-plane loads acting on the plate's edges have same shapes, enlarging the geometrical aspect ratio leads the plate loses its stability in wider ranges.

\section{References}

[1] D. L. Prabhakara and P. K. Datta, Vibration and static stability characteristics of rectangular plates with a localized flaw, Computers \& Structures, 49 (5) (1993) 825-836

[2] D. L. Prabhakara and P. K. Datta, Static and dynamic elastic behaviour of damaged plates subjected to local inplane loads, Marine Structures, 9 (8) (1996) 811-818

[3] D. L. Prabhakara and P. K. Datta, Vibration, buckling and parametric instability behaviour of plates with centrally located cutouts subjected to inplane edge loading (Tension or compression), Thin-Walled Structures, 27 (4) (1997) 287-310 
[4] P. J. Deolasi and P. K. Datta, Parametric instability characteristics of rectangular plates subjected to localized edge loading (compression or tension), Computers \& Structures, 54 (1) (1995) 73-82

[5] P. J. Deolasi and P. K. Datta, Simple and combination resonances of rectangular plates subjected to non-uniform edge loading with damping, Engineering Structures, 19 (12) (1997) 1011-1017

[6] K. N. Saha, R. C. Kar, and P. K. Datta, Dynamic stability of a rectangular plate on non-homogeneous winkler foundation, Computers \& Structures, 63 (6) (1997) 1213-1222

[7] S. K. Sahu and P. K. Datta, Parametric Instability of Doubly Curved Panels Subjected to Non-Uniform Harmonic Loading, Journal of Sound and Vibration, 240 (1) (2001) 117-129

[8] S. K. Sahu and P. K. Datta, Dynamic Stability of Curved Panels With Cutouts, Journal of Sound and Vibration, 251 (4) (2002) 683-696

[9] L. R. Kumar, P. K. Datta, and D. L. Prabhakara, Dynamic instability characteristics of laminated composite plates subjected to partial follower edge load with damping, International Journal of Mechanical Sciences, 45 (9) (2003) 1429-1448

[10] L. R. Kumar, P. K. Datta, and D. L. Prabhakara, Tension buckling and dynamic stability behaviour of laminated composite doubly curved panels subjected to partial edge loading, Composite Structures, 60 (2) (2003) 171181

[11] A. K. L. Srivastava, P. K. Datta, and A. H. Sheikh, Dynamic instability of stiffened plates subjected to non-uniform harmonic in-plane edge loading, Journal of Sound and Vibration, 262 (5) (2003) 1171-1189

[12] L. Ravi Kumar, P. K. Datta, and D. L. Prabhakara, Tension buckling and parametric instability characteristics of doubly curved panels with circular cutout subjected to nonuniform tensile edge loading, Thin-Walled Structures, 42 (7) (2004) 947-962

[13] L. Ravi Kumar, P. K. Datta, and D. L. Prabhakara, Dynamic instability characteristics of laminated composite doubly curved panels subjected to partially distributed follower edge loading, International Journal of Solids and Structures, 42 (8) (2005) 2243-2264

[14] S. N. Patel, P. K. Datta, and A. H. Sheikh, Buckling and dynamic instability analysis of stiffened shell panels, Thin-Walled Structures, 44 (3) (2006) 321-333

[15] R. I and S. C. Mohanty, Study on Free Vibration Analysis of Rectangular Plate Structures Using Finite Element Method, Procedia Engineering, 38 (2012) 2758-2766 
[16] B. C. L. Vanam, M. Rajyalakshmi, and R. Inala, Static analysis of an isotropic rectangular plate using finite element analysis (FEA), Journal of Mechanical Engineering Research, 4 (4) (2012) 148-162

[17] L. V. Kurpa and A. B. Linnik, Studying The Vibrations of In-Plane Loaded Plates of Variable Thickness, International Applied Mechanics, 41 (1) (2005) 62-69

[18] M. Bodaghi and A. R. Saidi, Buckling analysis of functionally graded Mindlin plates subjected to linearly varying in-plane loading using power series method of Frobenius, International Journal of Engineering, Transactions B: Applications, 25 (1) (2011) 89-106

[19] S. Hosseini-Hashemi, M. Fadaee, and S. R. Atashipour, A new exact analytical approach for free vibration of Reissner-Mindlin functionally graded rectangular plates, International Journal of Mechanical Sciences, 53 (2011) 11-22

[20] A. R. Pouladkhan, J. Emadi, and M. Safamehr, Numerical Study of Buckling of Thin Plates, World Academy of Science, Engineering and Technology, 78 (2011) 152-157

[21] A. J. Wilson and S. Rajasekaran, Elastic stability of all edges simply supported, stepped and stiffened rectangular plate under uniaxial loading, Applied Mathematical Modelling, 36 (2012) 5758-5772

[22] X. Wang and Y. Wang, Buckling analysis of thin rectangular plates under uniaxial or biaxial compressive point loads by the differential quadrature method, International Journal of Mechanical Sciences, 101-102 (2015) 3848

[23] Ć. B. Dolićanin, V. B. Nikolić, and D. Ć. Dolićanin. (2010) Application of Finite Difference Method to Study of the Phenomenon in the Theory of Thin Plates. Applied Mathematics, Informatics and Mechanics. 29-43

[24] A. C. Ugural, Stresses in Beams, Plates, And Shells, Third ed.: CRC Press, Taylor and Francis Group, 2011

[25] N. M. Werfalli and A. A. Karoud, Free Vibration Analysis of Rectangular Plates Using Galerkin-Based Finite Element Method, International Journal of Mechanical Engineering, 2 (2) (2012) 59-67

[26] A. S. Patil, Free Vibration Analysis of Thin Isotropic Rectangular Plate, International Journal of Innovative Research in Science, Engineering and Technology, 3 (4) (2014) 77-80

[27] J. C. Ezeh, O. M. Ibearugbulem, and C. I. Onyechere, Free-Vibration Analysis of Thin Rectangular Flat Plates Using Ordinary Finite Difference Method, Academic Research International, 4 (2) (2013) 187-192

[28] J. C. Ezeh, O. M. Ibearugbulem, and C. I. Onyechere, Pure Bending Analysis of Thin Rectangular Flat Plates Using Ordinary Finite Difference 
Method, International Journal of Emerging Technology and Advanced Engineering, 3 (3) (2013) 20-23

[29] S. S. Kolukula, Investigation of parametric instability in elastic structures, 2013

[30] A. W. Leissaa and J.-H. Kang, Exact solutions for vibration and buckling of an SS-C-SS-C rectangular plate loaded by linearly varying in-plane stresses, International Journal of Mechanical Sciences, 44 (2002) 19251945

[31] W. Chau Xie, Concept of Stability: Cambridge University Press, 2010

[32] P. Pedersen, Stability of the solutions to Mathieu-Hill equations with damping, Ingenieur-Archiv, 49 (1) (1980) 15-29

[33] E. Grebenikov, Y. A. Mitropolsky, and Y. A. Ryabov, Asymptotic Methods in Resonance Analytical Dynamics: CRC Press, 2004

[34] F. Verhulst, Methods and Applications of Singular Perturbations: Boundary Layers and Multiple Timescale Dynamics: Springer New York, 2006

[35] M. Bessa, Perturbations of Mathieu equations with parametric excitation of large period, Advances in Dynamical Systems and Applications, (2010)

[36] M. Gadella, H. Giacomini, and L. Lara, Periodic analytic approximate solutions for the Mathieu equation, Applied Mathematics and Computation, 271 (2015) 436-445 